\section{RSP}

http://www.rsp.fsp.usp.br/
Revista de Saúde Pública

\title{
Estimativa de custo da asma em tratamento ambulatorial: estudo com dados de mundo real
}

Eduardo Costa', Rosangela Caetano", Guilherme Loureiro Werneck"II, Maurício Bregman", Denizar Vianna Araújov", Rogério Rufino ${ }^{\mathrm{vI}}$

1 Universidade do Estado do Rio de Janeiro. Faculdade de Ciências Médicas. Departamento de Medicina Interna. Rio de Janeiro, RJ, Brasil

" Universidade do Estado do Rio de Janeiro. Instituto de Medicina Social. Departamento de Política, Planejamento e Administração em Saúde. Rio de Janeiro, RJ, Brasil

III Universidade do Estado do Rio de Janeiro. Instituto de Medicina Social. Departamento de Epidemiologia. Rio de Janeiro, RJ, Brasil

Iv Universidade do Estado do Rio de Janeiro. Faculdade de Ciências Médicas. Programa de Bolsas de Iniciação Científica. Rio de Janeiro, RJ, Brasil

$\checkmark$ Universidade do Estado do Rio de Janeiro. Faculdade de Ciências Médicas. Departamento de Clínica Médica. Rio de Janeiro, RJ, Brasil

vı Universidade do Estado do Rio de Janeiro. Faculdade de Ciências Médicas. Departamento de Doenças do Tórax. Rio de Janeiro, RJ, Brasil

\section{RESUMO}

OBJETIVO: Estimar o custo do diagnóstico e tratamento da asma.

MÉTODOS: Foi utilizada a perspectiva da sociedade. Foram incluídos por 12 meses em 2011-2012, sequencialmente, 117 indivíduos maiores de cinco anos de idade, em tratamento por asma nos Serviços de Pneumologia e Alergia-Imunologia da Policlínica Piquet Carneiro, Universidade do Estado do Rio de Janeiro. Todos realizaram duas entrevistas com seis meses de intervalo para coleta de dados, cobrindo 12 meses. As unidades de custos foram identificadas e valoradas de acordo com métodos definidos. Foi feita análise de sensibilidade e foram aplicados métodos estatísticos com nível de significância de 5\% para comparações de custos entre subgrupos.

RESULTADOS: Cento e oito pacientes completaram o estudo, 73,8\% eram mulheres. Mediana de idade foi de 49,5 anos. Rinite esteve presente em $83,3 \%$, e mais da metade tinha sobrepeso ou obesidade. A renda familiar média foi de $\mathrm{R} \$ 1.566,19 /$ mês $(\mathrm{DP}=1.503,30)$. A maioria dos trabalhadores e dos estudantes teve absenteísmo relacionado à asma. O custo médio anual total foi de $\mathrm{R} \$ 2.207,99 /$ paciente $(\mathrm{DP}=2.220,55)$. O custo relacionado à asma isolada foi de $\mathrm{R} \$ 1.984,17 /$ paciente-ano $(\mathrm{DP}=2.232,55)$. Asmáticos obesos, graves ou não controlados tiveram maiores custos em comparação aos não obesos $(p=0,001)$, não graves e controlados $(p=0,000)$. O custo direto correspondeu a $82,3 \%$ do custo total estimado. O custo com medicamentos para asma correspondeu a $62,2 \%$ dos custos diretos da asma.

CONCLUSÕES: Medicamentos para asma, medidas de controle ambiental e licenças de saúde prolongadas tiveram maior impacto potencial na variação do custo total. Os resultados são uma estimativa do custo do tratamento da asma em nível secundário no Sistema Único de Saúde, assumindo-se que o tratamento utilizado represente a abordagem ideal da doença.

DESCRITORES: Asma, economia. Antiasmáticos, provisão \& distribuição. Licença Médica. Custos de Cuidados de Saúde. Custos e Análise de Custo. Assistência Ambulatorial. Tomada de Decisão Clínica. 


\section{INTRODUÇÃO}

A asma é uma doença heterogênea definida pela ocorrência de sintomas variáveis e intermitentes. É caracterizada por inflamação crônica da via aérea associada à limitação variável do fluxo aéreo. A prevalência da asma aumentou no mundo desenvolvido nas últimas décadas do século XX e está frequentemente associada à rinite crônica ${ }^{6, a}$.

O estudo internacional de alergias e asma na infância (The International Study of Allergies and Asthma in Childhood [ISAAC]) encontrou prevalência média de 12,6\% e 14,6\% de rinite alérgica em crianças e adolescentes no Brasil, respectivamente, e prevalência de asma de $24,3 \%$ e $19,0 \%$ nos mesmos grupos ${ }^{24}$. Estudo apontou prevalência de 13,1\% de asma em adolescentes brasileiros em $2013-2014^{15}$ e dados da região sul do país apontaram prevalência de asma em adultos de 5,2\% em 2010 . Estudo latino-americano com base em inquérito telefônico estimou prevalência da asma no Brasil de $13,3 \%{ }^{12}$. As últimas diretrizes nacionais em asma sugerem a existência de 20 milhões de asmáticos no país ${ }^{23}$.

A asma tem custo significativo para os sistemas de saúde em todo o mundo em virtude da elevada prevalência. Estudo analisou dados de 47.033 adultos norte-americanos atendidos no sistema de saúde entre 2003 e 2005 e estimou gasto médico total no país de US\$18 bilhões ${ }^{26}$. Van Den Akker-van Marle et al. estimaram custo anual direto total para a asma e sibilância infantil de $€ 3$ milhões nos 25 países europeus participantes, chegando a $€ 5,2$ milhões quando projetado para toda a União Europeia (UE) ${ }^{28}$. Para adultos, o custo médio anual foi de $€ 1.583 /$ paciente, com um custo total estimado de $€ 4,3$ bilhões para os 11 países estudados e de $€ 19,3$ bilhões, ao se projetar para toda a região 2 . O custo total com a doença na região Ásia-Pacífico foi equivalente a 13,0\% do produto interno bruto (PIB) da região, enquanto nos EUA correspondeu a 2,0\% ${ }^{26}$. Entretanto, as diferentes metodologias empregadas nos estudos não permitem uma comparação direta dessas proporções entre os países.

Os dados sobre custos da asma no Brasil são limitados, com poucos estudos publicados e abordam principalmente casos graves, pelo seu impacto econômico decorrente da maior utilização de recursos hospitalares ${ }^{13,22}$. No entanto, a Organização Mundial da Saúde (OMS) estima que apenas 5,0\% a 10,0\% dos asmáticos tenham a forma grave da doença ${ }^{a}$. Felizmente, reduziu o número de internações por asma no Sistema Único de Saúde (SUS) nos últimos anos, e esse resultado positivo provavelmente reflete o maior uso de corticóides inalatórios devido à sua dispensação gratuita no SUS ${ }^{\mathrm{b}}$. Entretanto, dados de custo da asma em tratamento ambulatorial abordando diferentes níveis de gravidade e controle da doença são escassos em nosso meio.

O objetivo deste estudo foi estimar os custos associados ao diagnóstico e tratamento da asma em cenário de mundo real. Esses dados podem auxiliar a decisão de formuladores de políticas de saúde na alocação de recursos para pesquisa e assistência a asmáticos no SUS, considerando uma abordagem tida como ideal para a doença.

${ }^{a}$ Global Initiative for Asthma. 2017 GINA Report. Global strategy for asthma management and prevention; 2017 [citado 1 dez 2017]. Disponível em: http://ginasthma.org/2017-ginareport-global-strategy-for-asthmamanagement-and-prevention/

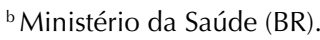
Medicamentos gratuitos para asma ajudaram a reduzir em $16 \%$ as internações. Brasília (DF); 2014 [citado 3 nov 2017]. Disponível em: http://www. brasil.gov.br/saude/2013/06/ medicamentos-gratuitospara-asma-ajudam-a-reduzirinternacoes-no-sus

\section{MÉTODOS}

Estudo de custo de doença, com abordagem baseada na prevalência da asma e tomando por base a perspectiva da sociedade. Foram coletados dados referentes a custos diretos e indiretos. As unidades de custo e suas quantidades foram estimadas a partir da coleta de dados primários.

Foram incluídos pacientes com até seis anos de idade, com diagnóstico clínico e funcional de asma persistente (de acordo com critérios estabelecidos) ) $^{23, a} \mathrm{em}$ acompanhamento nos serviços especializados em asma (alergia-imunologia e tisio-pneumologia) de uma unidade ambulatorial universitária localizada na cidade do Rio de Janeiro, RJ, de abril a outubro de 2011. Foram excluídos pacientes que abandonaram o tratamento (sem retorno por período maior que quatro meses) e aqueles com doença crônica que poderia causar sintomas respiratórios semelhantes à asma. 
Os pacientes realizaram visitas clínicas de rotina com três a quatro meses de intervalo e duas entrevistas para coleta de dados com intervalo de seis meses, realizadas por dois profissionais que não participaram da assistência. Os dados coletados englobaram 12 meses (abril/2011 a março/2012), cobrindo todas as estações climáticas. Foi utilizado instrumento estruturado e padronizado, previamente submetido a pré-teste. A entrevista foi respondida pelos responsáveis dos pacientes menores de 18 anos.

As variáveis primárias coletadas relacionaram-se ao custo de visitas de rotina (com médico, enfermeiro ou fisioterapeuta) e de urgência, dias de internação, exames complementares, medicamentos usados para asma, rinite e infecções respiratórias (IR), uso de imunoterapia (IMTP), gastos com transporte, medidas de controle de aeroalérgenos (higiene ambiental [HA]), horas perdidas de trabalho dedicadas ao tratamento e número de dias de absenteísmo no trabalho e escola. Para se evitar viés de memória, os dados referentes aos três meses anteriores à coleta foram duplicados para se estimar os gastos do semestre. Foram excluídos o número de dias ou local de hospitalização e os gastos com HA, que eram facilmente lembrados em relação ao período completo. A estimativa do custo indireto foi obtida por meio da abordagem do capital humano, visto que a população estudada tinha grande heterogeneidade de ocupações e esse método mostra-se adequado. As unidades de custo, fontes e o método de sua valoração, por microcusteio, estão definidos na Quadro. Todos foram considerados como custos exclusivos da asma, subtraídos os custos com medicamentos para rinite e para infecções respiratórias (IR). Variáveis secundárias foram idade, sexo, escolaridade, renda familiar mensal (RFM), índice de massa corporal (IMC), gravidade e controle da asma. A gravidade e o controle da asma foram classificados de acordo com a GINA ${ }^{\text {a }}$. Sobrepeso e obesidade foram definidos pelos critérios da OMS9.

Todas as variáveis econômicas foram quantificadas em reais. Para comparação com dados internacionais, os valores foram convertidos para dólares americanos pela paridade do poder de compra (PPC) em 2012 (US\$1,00 = R \$1,71) $)^{c}$. As médias anuais de custo da asma foram estratificadas por gênero, faixa etária, RFM, gravidade e controle da asma, estado nutricional (normal, sobrepeso ou obesidade) e presença de rinite. $O$ teste do qui-quadrado (com correção de Fisher, quando necessário) e o teste de Mann-Whitney foram utilizados para comparar as diferenças entre as variáveis categóricas e entre variáveis contínuas, respectivamente. As diferenças entre subgrupos por níveis de gravidade, controle e estado nutricional foram analisadas por métodos de regressão bivariada e múltipla. Na análise de sensibilidade, devido à grande dispersão de dados, utilizamos os valores dos percentis 25-75 $\left(\mathrm{P}_{25-75}\right)$ em relação à mediana das unidades de custo (consultas, hospitalização, exames complementares, medicamentos para asma, rinite e IR, IMTP, transporte, higiene ambiental, absentismo e benefício auxílio doença [BAD]) para o cálculo do impacto da variação de cada uma delas no custo total.

Os dados foram inseridos em planilhas Microsoft ${ }^{\circledR}$ Office Excel 2010 (Microsoft Corp., CA, USA) para análise descritiva das características gerais e de custos. Para a análise bivariada, foi empregado o programa GraphPad Prism 6.0 (GraphPad Software Inc., CA, USA). Para análise múltipla, foi feita regressão logística com transformação logarítmica utilizando o pacote Stata 11 (Stata Corp, TX, USA). Foi adotado nível de significância de 5\%.

Os pacientes (ou seus responsáveis) assinaram termo de consentimento livre e esclarecido. O estudo foi aprovado pelo Comitê de Ética em Pesquisa local e foi registrado no Comitê Nacional de Ética em Pesquisa (CEP/HUPE 2904/2011 - CAAE 0054.0.228.000-11).

${ }^{\mathrm{c}}$ The World Bank. PPP conversion factor, private consumption (LCU per international \$); 2014 [citado 1 dez 2017]. Disponível em: https://data.worldbank. org/indicator/PA.NUS. $\mathrm{PP}$ ? locations $=\mathrm{BR}$

\section{RESULTADOS}

Foram incluídos 117 pacientes e 7,7\% não completaram o acompanhamento. A população estudada $(n=108)$ representava $33,1 \%$ dos pacientes asmáticos em tratamento na unidade durante o ano, 73,2\% era do sexo feminino, com predomínio de adultos e mediana de idade de 49,5 anos $\left(\mathrm{P}_{25-75}=27,7-60,0\right)$. A maioria $(70,4 \%)$ completou o ensino fundamental, $23,1 \%$ 
Quadro. Unidades de custo da asma em tratamento ambulatorial e métodos de valoração.

\begin{tabular}{|c|c|}
\hline \multicolumn{2}{|c|}{$\begin{array}{l}\text { Consultas (médicas, de enfermagem ou fisioterapia), atendimento de urgência, hospitalizações (quarto, enfermaria ou terapia intensiva) } \\
\text { e exames complementares }\end{array}$} \\
\hline Sistema Único de Saúde (SUS) & Sistema de informações ambulatoriais e hospitalares do SUS - SIA e SIH/SIGTAP/Datasus ${ }^{a}$ \\
\hline Sistema de Saúde Suplementar (SSS) & Classificação brasileira hierarquizada de procedimentos médicos (CBHPM) - versão $2012^{\mathrm{b}}$ \\
\hline Sistema privado & Valor informado pelo paciente/responsável \\
\hline \multicolumn{2}{|c|}{ Medicamentos para asma, rinite e infecções respiratórias } \\
\hline Fornecidos pelo SUS & $\begin{array}{l}\text { Tabela de preços de medicamentos para compras públicas da Secretaria Executiva da } \\
\text { Agência Nacional de Vigilância Sanitária (Anvisa), atualizada em março/2012 }\end{array}$ \\
\hline Financiados pelas famílias & $\begin{array}{c}\text { Tabela de preços de medicamentos para venda com preço máximo ao consumidor da } \\
\text { Secretaria Executiva da Agência Nacional de Vigilância Sanitária (Anvisa), atualizada em } \\
\text { março/2012c. Foi utilizada a média de preço de três formulações diferentes para cada } \\
\text { medicamento utilizado }\end{array}$ \\
\hline \multicolumn{2}{|l|}{ Imunoterapia (vacinas para alergia) } \\
\hline Frasco & Valor de aquisição informado pela Divisão Financeira da Unidade - abril de 2012 \\
\hline Aplicação (todas em unidades SUS) & Sistema de informações ambulatoriais e hospitalares do SUS - SIA e SIH/SIGTAP/Datasus ${ }^{a}$ \\
\hline \multicolumn{2}{|r|}{ Medidas de controle ambiental } \\
\hline $\begin{array}{l}\text { Itens adquiridos (capas, cortinas, filtros de ar) e despesas } \\
\text { para obras no piso, pintura e mudança de móveis }\end{array}$ & Valor informado pelo paciente/responsável \\
\hline \multicolumn{2}{|c|}{ Transporte à unidade de saúde para atendimento e exames } \\
\hline Transporte coletivo ou táxi & Valor informado pelo paciente/responsável \\
\hline Veículo privado ou veículos de assistência social do SUS & $\begin{array}{c}\text { O custo foi estimado utilizando o valor de } 1 \text { litro de gasolina para cada } 10 \mathrm{~km} \text { de distância } \\
\text { percorridos. As distâncias entre o ponto de partida e o destino foram obtidas no portal de } \\
\text { internet Goog/e Maps }\end{array}$ \\
\hline \multicolumn{2}{|r|}{ Absenteísmo } \\
\hline Escola & Número de dias (não valorado) \\
\hline Trabalho (licenças até 15 dias) & $\begin{array}{l}\text { Valor de cada dia }=\text { renda mensal informada do indivíduo/21 dias. Custo }=\text { valor de cada } \\
\qquad \text { dia } x \text { número de dias perdidos }\end{array}$ \\
\hline $\begin{array}{l}\text { Trabalho (Benefício auxílio doença [BAD] licenças > } 15 \\
\text { dias) }\end{array}$ & 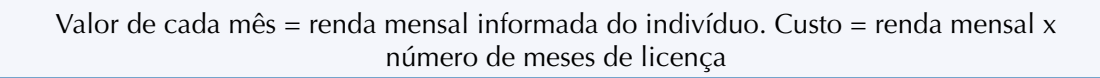 \\
\hline
\end{tabular}

SUS: sistema único de saúde; SAI: sistema de informações ambulatoriais; SIH: sistema de informações hospitalares; SIGTAP: sistema de gerenciamento da tabela de procedimentos, medicamentos e OPM (órteses, próteses e materiais) do SUS; Datasus: departamento de informática do SUS; SSS: sistema de saúde suplementar; CBHPM: classificação brasileira hierarquizada de procedimentos médicos

a Tabela de procedimentos - ambulatório e internação SIA e SIH - SUS; 2014 [citado 1 dez 2017]. Disponível em: http://sigtap.datasus.gov.br/tabelaunificada/app/sec/inicio.jsp

b Classificação brasileira hierarquizada de procedimentos médicos (CBHPM); 2012 [citado 21 out 2014]. Disponível em http://www.acm.org.br/ cbhpm-2012/

c Agência Nacional de Vigilância Sanitária (Anvisa - BR). Brasília (DF). Tabela de preços de medicamentos para compras públicas da Secretaria Executiva da Anvisa; 2012 [citado 21 out 2014]. Disponível em: http://portal.anvisa.gov.br/cmed

${ }^{d}$ Google. Google Maps. Distâncias em KM entre o ponto de partida (residência do paciente) e o destino (unidade de saúde); 2014 [citado 1 dez 2017]. Disponível em: https://www.google.com.br/maps/place/Av.+Mal.+Rondon,+381+-+S\%C3\%A3o+Francisco+Xavier,+Rio+de+Janeiro+-+RJ,+20950-003/@22.9061884,-43.2795421,13z/data=!4m5!3m4!1s0x997e7d1d6deb6f:0x43bf435b497372f9!8m2!3d-22.9061884!4d-43.2445232

e Agência Nacional do Petróleo, Gás Natural e Biocombustíveis (ANP - BR) - Rio de Janeiro (RJ); 2012 [citado 1 dez 2017]. Série histórica do levantamento de preços e de margens de comercialização de combustíveis. Disponível em: http://anp.gov.br/wwwanp/precos-e-defesa-da-concorrencia/ precos/levantamento-de-precos/serie-historica-do-levantamento-de-precos-e-de-margens-de-comercializacao-de-combustiveis

o ensino médio e 6,5\% o ensino superior. A RFM foi de R $\$ 1.566,19$ (DP = 1.503,30). A RFM foi menor entre pacientes com asma não controlada em comparação com controle parcial ou total nos dois semestres ( $\mathrm{p}=0,005 \mathrm{e} \mathrm{p}=0,01$, respectivamente). A mediana de duração da asma foi de 18 anos $\left(\mathrm{P}_{25-75}=9,5-33,0\right)$. Na época da inclusão no estudo, 49,1\% dos pacientes tinham asma classificada como leve, $36,1 \%$ moderada e 14,8\% grave. Na avaliação final, a classificação de $38,9 \%$ dos pacientes mudou para asma intermitente e $38,1 \%$ dos $21 \mathrm{com}$ asma não controlada obtiveram melhor controle. Setenta e quatro pacientes (68,5\%) tinham sobrepeso ou obesidade. A rinite estava presente em $83,0 \%$ dos pacientes e $28,0 \%$ utilizaram antibióticos para tratamento de IR durante o estudo.

Houve grande variação do desvio-padrão em relação à média dos valores de algumas unidades de custo, apontando para a grande dispersão nesses itens (Tabela 1). O custo da asma foi significantemente maior em pacientes sem rinite $(p=0,03)$, em adultos $(p=0,000)$, assim 
Tabela 1. Resultados da valoração das unidades de custo da asma em tratamento ambulatorial - totais, médias e medianas no $1^{\circ}$ e $2^{\circ}$ período de estudo.

\begin{tabular}{|c|c|c|c|c|c|c|c|c|c|c|}
\hline \multirow{2}{*}{ Unidades de custo } & \multicolumn{2}{|c|}{$1^{\circ}$ período } & \multicolumn{2}{|c|}{$2^{\circ}$ período } & \multirow{2}{*}{$\begin{array}{c}\text { Total } \\
\mathbf{R} \$\end{array}$} & \multirow{2}{*}{$\frac{\text { Média }^{\mathrm{a}}}{\mathrm{R} \$}$} & \multirow{2}{*}{$\begin{array}{l}\text { DP } \\
\text { R\$ }\end{array}$} & \multirow{2}{*}{$\frac{\text { Mediana }^{\text {b }}}{\text { R\$ }}$} & \multirow{2}{*}{$\begin{array}{c}\text { Perc25 } \\
\text { R\$ } \\
\end{array}$} & \multirow{2}{*}{$\begin{array}{c}\text { Perc75 } \\
\text { R\$ } \\
\end{array}$} \\
\hline & $\mathbf{n}$ & $\mathbf{R} \$$ & $\mathbf{n}$ & $\mathbf{R} \$$ & & & & & & \\
\hline \multicolumn{11}{|l|}{ Custos diretos } \\
\hline Visitas médicas agendadas & 444 & $5.160,00$ & 331 & $3.397,00$ & $8.557,00$ & 79,23 & 92,04 & 60,00 & 60,00 & 80,00 \\
\hline $\begin{array}{l}\text { Visitas médicas não } \\
\text { agendadas/Urgência }^{c}\end{array}$ & 213 & $3.778,48$ & 99 & $1.368,06$ & $5.146,54$ & 47,65 & 148,63 & 49,88 & 24,94 & 99,76 \\
\hline $\begin{array}{l}\text { Atendimento de } \\
\text { enfermagem/Fisioterapia }^{\mathrm{d}}\end{array}$ & 216 & $1.078,20$ & 202 & $1.002,28$ & $2.080,48$ & 19,26 & 47,05 & 44,10 & 37,80 & 53,55 \\
\hline Visitas (total) & 873 & $10.048,68$ & 632 & $5.685,14$ & $15.733,82$ & 145,68 & 230,98 & 97,80 & 60,00 & 151,57 \\
\hline Hospitalização (dias) & 13 & $1.298,88$ & 7 & 865,92 & $2.164,80$ & 20,04 & 108,89 & 432,96 & 432,96 & 541,20 \\
\hline Exames complementares & 832 & $5.357,44$ & 466 & $3.820,74$ & $9.178,18$ & 84,98 & 127,54 & 43,62 & 29,84 & 79,08 \\
\hline $\begin{array}{l}\text { Medicamentos para controle } \\
\text { da asma (doses) }\end{array}$ & 32.374 & $45.775,94$ & 36.204 & $43.569,42$ & $89.345,36$ & 827,27 & 659,58 & 799,81 & 426,09 & $1.084,32$ \\
\hline $\begin{array}{l}\text { Medicamentos para resgate } \\
\text { da asma (doses) }\end{array}$ & 164.962 & $9.568,72$ & 96.484 & $7.598,82$ & $17.167,54$ & 158,96 & 420,07 & 47,94 & 9,07 & 129,23 \\
\hline $\begin{array}{l}\text { Medicamentos para asma } \\
\text { (total) }\end{array}$ & 197.336 & $55.344,66$ & 132.688 & $51.168,24$ & $106.512,90$ & 986,23 & 978,94 & 825,37 & 428,40 & $1.229,11$ \\
\hline $\begin{array}{l}\text { Medicamentos para rinite } \\
{(\text { doses })^{\mathrm{e}}}\end{array}$ & 40.442 & $7.412,90$ & 33.448 & $9.237,94$ & $16.650,84$ & 154,17 & 230,14 & 145,87 & 57,52 & 232,82 \\
\hline $\begin{array}{l}\text { Medicamentos para } \\
\text { infecção respiratória (doses) }\end{array}$ & 2.058 & $7.430,12$ & 1.484 & 994,68 & $8.424,80$ & 78,01 & 213,14 & 180,00 & 79,80 & 272,00 \\
\hline $\begin{array}{l}\text { Imunoterapia/Vacinas para } \\
\text { alergia }(\text { doses+frascos) })^{g}\end{array}$ & 852 & $1.594,30$ & 468 & $1.688,32$ & $3.282,62$ & 30,39 & 53,70 & 91,40 & 79,15 & 121,89 \\
\hline Controle ambiental ${ }^{\mathrm{h}}$ & - & $10.285,60$ & - & $11.198,61$ & $21.484,21$ & 198,93 & 542,67 & 200,00 & 78,75 & 486,75 \\
\hline Transporte & - & $7.161,90$ & - & $5.627,96$ & $12.789,86$ & 118,42 & 194,46 & 75,64 & 51,95 & 129,55 \\
\hline \multicolumn{11}{|l|}{ Custos indiretos } \\
\hline Faltas ao trabalho (días) ${ }^{i}$ & 186 & $4.034,45$ & 94 & $4.026,00$ & $8.060,45$ & 74,63 & 185,60 & 174,97 & 66,60 & 359,86 \\
\hline Faltas a escola (dias) $)^{i}$ & 61 & - & 48 & - & - & - & - & - & - & - \\
\hline $\begin{array}{l}\text { Benefício auxílio-doença } \\
(\mathrm{BAD})^{\mathrm{k}}\end{array}$ & 4 & $24.800,00$ & 2 & $9.380,00$ & $34.180,00$ & 316,48 & $1.622,51$ & $4.500,00$ & $4.450,00$ & $9.850,00$ \\
\hline Custo total & - & $134.768,93$ & - & $103.693,55$ & $238.462,48$ & $2.207,99$ & $2.220,55$ & $1.634,39$ & $1.133,76$ & $2.092,12$ \\
\hline Custo relacionado a asma & - & $119.925,91$ & - & $93.460,93$ & $213.386,84$ & $1.975,80$ & $2.232,55$ & $1.414,96$ & 858,92 & $1.924,71$ \\
\hline $\begin{array}{l}\text { Custo relacionado a rinite e } \\
\text { infecção respiratória }\end{array}$ & - & $14.843,02$ & - & $10.232,62$ & $25.075,64$ & 232,18 & 319,79 & 175,99 & 87,30 & 345,25 \\
\hline
\end{tabular}

a Calculada em relação ao total da população estudada $(n=108)$.

${ }^{\mathrm{b}}$ Calculada em relação ao número de indivíduos que geraram a unidade de custo em questão.

${ }^{c} \mathrm{n}=43$ indivíduos.

${ }^{\mathrm{d}} \mathrm{n}=33$ indivíduos.

e $\mathrm{n}=84$ indivíduos.

${ }^{\mathrm{f}} \mathrm{n}=31$ indivíduos.

${ }^{\mathrm{g}} \mathrm{n}=32$ indivíduos.

${ }^{\mathrm{h}} \mathrm{n}=54$ indivíduos.

i $\mathrm{n}=32$ indivíduos.

i $\mathrm{n}=18$ indivíduos.

${ }^{k} \mathrm{n}=5$ indivíduos/ 6 benefícios auxílio-doença (BAD).

como nos pacientes mais graves $(p=0,000)$, com pior controle $(p=0,000)$ e maior peso $(\mathrm{p}=0,001)$. O custo da medicação para asma também foi maior nos pacientes com duração $>20$ anos de doença $(p=0,008)$ (Tabela 2). Os custos diretos representaram $82,3 \%$ do custo total, $49,7 \%$ dos custos exclusivos da asma corresponderam a custos com medicamentos, equivalentes a $62,2 \%$ dos custos diretos exclusivos da asma (Tabela 1 ).

Três pacientes foram hospitalizados devido à asma (média de 4,3 dias/paciente) nos primeiros seis meses, e dois (média de 3,5 dias/paciente) o foram no semestre final do estudo. Todas as hospitalizações ocorreram no hospital da universidade, no SUS.

Dezoito dos 24 estudantes ausentaram-se da escola e 28 pacientes faltaram ao trabalho por causa da asma. Incluindo os quatro que faltaram ao trabalho para cuidar de seus filhos, 
32 trabalhadores (78,0\% dos indivíduos que exerciam atividades laborativas extradomiciliares) ausentaram-se por motivos relacionados à asma. Na primeira avaliação, quatro pacientes estavam recebendo benefício de auxílio-doença (BAD). Na segunda, dois destes tinham sido aposentados por causa da asma, um perdeu o emprego e dois continuavam em BAD. O custo anual estimado relacionado ao absentismo foi de $\mathrm{R} \$ 42.240,45$, a maior proporção devida à $\mathrm{BAD}$ (Tabela 1).

A maior gravidade e o pior controle da asma se confirmaram como fatores independentes associados ao maior custo da doença. Houve tendência à influência do sexo como fator independente, assim como o peso isoladamente não se mostrou um fator independente da gravidade e do controle no resultado final do custo (Tabela 3). O diagrama de Tornado (Figura) apresenta a variação do custo total baseada nos percentis 25-75 em torno da mediana das diferentes unidades de custo. Mostra o importante impacto potencial das variações dos custos associados aos medicamentos para asma, medidas para controle ambiental, licenças prolongadas (BAD) e medicamentos para rinite sobre o custo total da doença.

Tabela 2. Custo médio anual da asma em tratamento ambulatorial, estratificado por características demográficas e clínicas.

\begin{tabular}{|c|c|c|c|c|c|c|c|c|c|}
\hline \multirow{2}{*}{ Categoria } & & \multirow{2}{*}{ n (\%) } & \multicolumn{2}{|c|}{ Total } & \multicolumn{2}{|c|}{ Asma } & \multicolumn{2}{|c|}{ Medicamentos asma } & \multirow{2}{*}{$\mathbf{p}$} \\
\hline & & & Média & DP & Média & DP & Média & DP & \\
\hline \multirow{3}{*}{ Idade (anos) } & $<20$ & $21(19,4)$ & $1.273,26$ & 300,65 & $1.028,70$ & 270,79 & 401,08 & 187,45 & $0,000^{\mathrm{a}}$ \\
\hline & $20-59$ & $61(56,5)$ & $2.709,30$ & $1.653,10$ & $2.498,65$ & $1.649,24$ & $1.184,45$ & 626,10 & $0,000^{\mathrm{b}}$ \\
\hline & $\geq 60$ & $26(24,1)$ & $1.683,78$ & 492,00 & 826,93 & 442,10 & $1.028,57$ & 306,86 & \\
\hline \multirow{2}{*}{ Gênero } & Masculino & $29(26,8)$ & $2.604,31$ & $1.762,99$ & $1.422,85$ & $1.765,51$ & 947,25 & 568,78 & $0,52^{\mathrm{a}}$ \\
\hline & Feminino & $79(73,2)$ & $2.028,61$ & $1.089,65$ & $1.039,80$ & $1.075,91$ & $1.011,98$ & 504,77 & $0,40^{\mathrm{b}}$ \\
\hline \multirow{3}{*}{ Renda familiar } & $\leq 1$ salário mínimo & $25(23,1)$ & $2.249,88$ & 946,69 & $1.213,02$ & 958,39 & $1.250,63$ & 664,03 & $0,58^{a}$ \\
\hline & > 1 a 3 salários mínimos & $53(49,1)$ & $2.120,08$ & $1.290,25$ & $1.089,94$ & $1.283,95$ & 897,37 & 459,03 & $0,38^{\mathrm{a}}$ \\
\hline & > 3 salários mínimos & $30(27,8)$ & $2.254,43$ & $1.572,65$ & $1.190,75$ & $1.558,36$ & 985,37 & 504,07 & \\
\hline \multirow{3}{*}{ Escolaridade } & Fundamental & $76(70,4)$ & $1.991,02$ & $1.013,29$ & $1.045,56$ & $1.012,15$ & 952,30 & 451,06 & $0,92^{\mathrm{a}}$ \\
\hline & Média & $25(23,1)$ & $2.761,38$ & $1.934,25$ & $1.414,95$ & $1.925,73$ & 997,65 & 612,42 & $0,55^{\mathrm{b}}$ \\
\hline & Superior & $7(6,5)$ & $2.204,65$ & $1.334,30$ & $1.224,35$ & $1.351,14$ & $1.442,88$ & 814,20 & \\
\hline \multirow{3}{*}{ Duração asma (anos) } & $\leq 10$ & $36(33,4)$ & $1.107,11$ & $1.492,66$ & 590,86 & $1.496,80$ & 439,32 & 549,42 & $0,30^{\mathrm{a}}$ \\
\hline & $>10-20$ & $26(24,1)$ & $1.030,48$ & $1.050,13$ & 503,71 & $1.023,44$ & 458,81 & 522,03 & $0,008^{b}$ \\
\hline & $>20$ & $46(42,5)$ & $1.114,01$ & $1.294,13$ & 596,26 & $1.286,16$ & 564,44 & 496,41 & \\
\hline \multirow{4}{*}{ Gravidade da asmac $^{c}$} & Intermitente & $42(38,9)$ & 738,93 & 833,01 & 383,21 & 807,65 & 276,35 & 222,93 & \\
\hline & Leve & $24(22,2)$ & 766,66 & 456,98 & 377,44 & 406,67 & 372,52 & 278,75 & $0,000^{\mathrm{a}}$ \\
\hline & Moderada & $31(28,7)$ & $1.121,90$ & $1.519,51$ & 591,50 & $1.515,62$ & 484,12 & 293,85 & $0,000^{\mathrm{b}}$ \\
\hline & Grave & $11(10,2)$ & $2.400,57$ & $2.018,69$ & $1.352,15$ & $2.025,91$ & $1.283,17$ & 975,16 & \\
\hline \multirow{3}{*}{ Controle da asma ${ }^{c}$} & Controlada & $53(49,1)$ & 717,31 & 300,21 & 362,72 & 293,54 & 330,27 & 202,86 & $0,000^{a}$ \\
\hline & Parcialmente controlada & $42(38,9)$ & $1.090,19$ & $1.270,55$ & 557,56 & $1.262,29$ & 480,10 & 362,52 & $0,000^{\mathrm{b}}$ \\
\hline & Não controlada & $13(12,0)$ & $2.195,21$ & $2.095,52$ & $1.220,64$ & $2.110,98$ & $1.044,91$ & 948,45 & \\
\hline \multirow{3}{*}{ Estado nutricional } & Normal & $44(40,7)$ & $1.757,33$ & $1.071,37$ & 873,37 & $1.042,72$ & 681,98 & 274,66 & $0,001^{\mathrm{a}}$ \\
\hline & Sobrepeso & $35(32,4)$ & $2.154,48$ & 983,88 & $1.155,63$ & 968,13 & $1.070,78$ & 550,83 & $0,000^{b}$ \\
\hline & Obesidade & $29(26,9)$ & $2.864,01$ & $1.824,36$ & $1.535,55$ & $1.842,37$ & $1.376,96$ & 683,37 & \\
\hline \multirow{2}{*}{ Rinite } & Sim & $90(83,3)$ & $1.087,66$ & $1.282,21$ & 557,45 & $1.275,87$ & 446,70 & 478,56 & $0,03^{a}$ \\
\hline & Não & $18(16,7)$ & $1.111,24$ & $1.442,09$ & 645,85 & $1.433,20$ & 750,26 & 648,76 & $0,0001^{b}$ \\
\hline
\end{tabular}

SM: salário mínimo

${ }^{a}$ Comparações relativas ao custo da asma isolada.

${ }^{\mathrm{b}}$ Comparações relativas ao custo de medicamentos para asma.

${ }^{\mathrm{c}}$ Comparações relativas ao controle e gravidade da asma na $2^{\mathrm{a}}$ e $1^{\mathrm{a}}$ avaliação. 
Tabela 3. Análise multivariada para fatores independentes sobre o custo da asma em tratamento ambulatorial.

\begin{tabular}{lcc}
\hline Variável & Coeficiente $($ IC95\%) & p \\
\hline Idade & $-0,0003862(-0,006028-0,0052556)$ & 0,89 \\
Gênero & $0,2406721(0,0070797-0,4742645)$ & 0,04 \\
Escolaridade & $-0,0975651(-0,3368083-0,141678)$ & 0,42 \\
Renda mensal & $0,0012074(-0,0997113-0,102126)$ & 0,98 \\
Peso & $-0,2074344(-0,423744-0,0088752)$ & 0,06 \\
Gravidade da asma & $0,1637397(0,0322054-0,295274)$ & $\mathbf{0 , 0 1}$ \\
Controle da asma & $0,6330797(0,288369-0,9777903)$ & $\mathbf{0 , 0 0 0}$ \\
\hline
\end{tabular}

Os valores de p em negrito indicam os fatores independentes sobre o custo da asma.

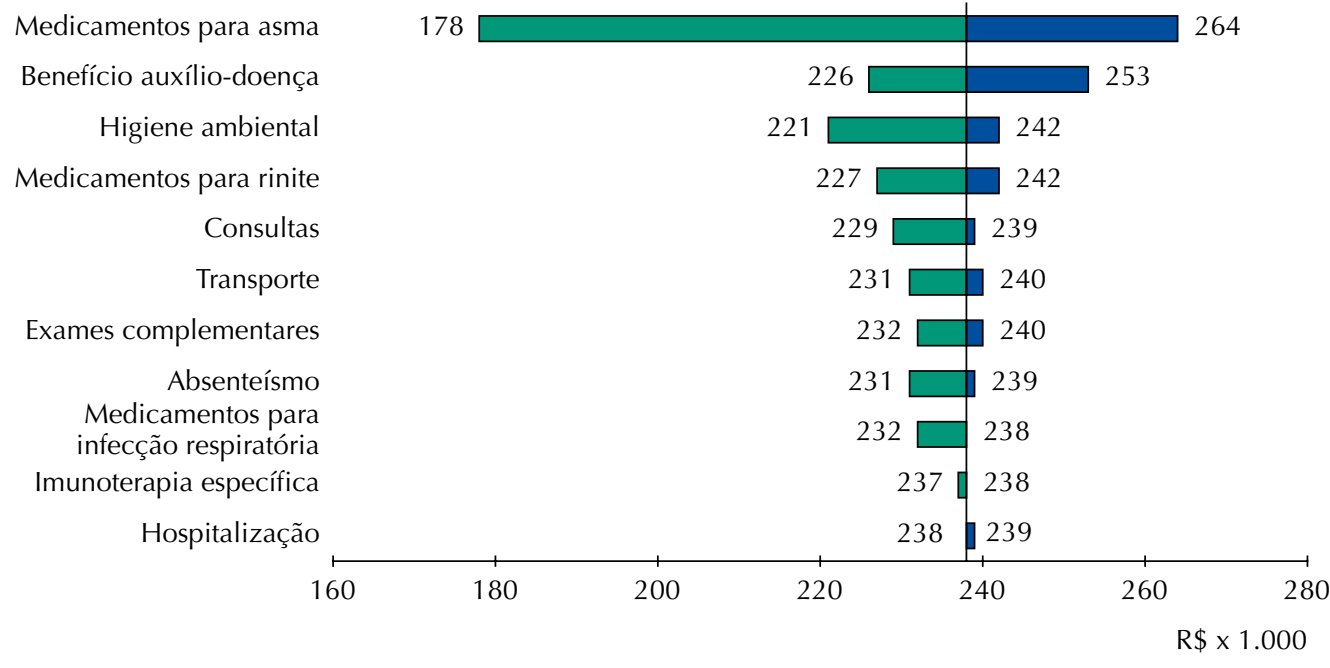

Figura. Análise de sensibilidade para variações das diferentes unidades de custo da asma em tratamento ambulatorial (diagrama de Tornado).

\section{DISCUSSÃO}

Poucos estudos com dados primários sobre custos de doenças crônicas estão publicados no Brasil e, em sua maioria, referem-se a doenças cardiovasculares como doença arterial coronariana crônica ${ }^{20} \mathrm{e}$ insuficiência cardíaca congestiva ${ }^{3}$. Revisão sistemática recente incluiu apenas três estudos sobre custos da asma no Brasil, todos com foco apenas na asma grave, mostrando a escassez de dados em nosso país ${ }^{25}$. Nosso estudo traz uma estimativa de custo de asma brônquica em um cenário de vida real, com pacientes ambulatoriais e diferentes níveis de gravidade e controle, ainda não descrita em nosso meio.

Do ponto de vista clínico, a abordagem utilizada resultou em mudanças desejáveis na classificação de gravidade dos pacientes, assim como melhoria do controle em parte deles.

Os diferentes métodos utilizados no tratamento e na valoração dos custos é um problema na comparação de resultados entre diferentes países. Considerando os valores ajustados pela PPC, o custo anual total estimado para a asma por paciente em nosso estudo foi 50,0\% maior do que a média da região Ásia-Pacífico ${ }^{16}$. Por outro lado, correspondeu a pouco mais da metade do custo estimado da asma nos Estados Unidos ${ }^{26} \mathrm{e}$ em adultos da UE ${ }^{2}$ (59,8\% e $55,0 \%$, respectivamente).

Asma e rinite estão frequentemente associadas e influenciam-se mutuamente. Estudos indicam que 75,0\% a 80,0\% dos asmáticos têm rinite e que 40,0\% a 50,0\% dos indivíduos com rinite alérgica ou eosinofílica não alérgica têm hiperresponsividade brônquica ${ }^{a}$. Em nosso estudo, a coexistência de asma e rinite foi alta e o custo exclusivo da asma foi maior nos 
dThe World Bank. GDP per capita (current US\$; 2014) [citado 1 dez 2017]. Disponível em: https://data.worldbank.org/ indicator/NY.GDP.PCAP.CD

e Ministério da Saúde (BR), Secretaria de Vigilância em Saúde, Departamento de Vigilância de Doenças e Agravos não Transmissíveis e Promoção da Saúde. Vigitel Brasil 2012: vigilância de fatores de risco e proteção para doenças crônicas por inquérito telefônico. Brasília (DF); 2013 [citado 3 nov 2017]. Disponível em: http://pesquisa. bvsalud.org/bvsms/resource/pt/ mis-37272

'Ministério da Saúde (BR), Secretaria de Vigilância em Saúde, Departamento de Vigilância de Doenças e Agravos não Transmissíveis e Promoção da Saúde. Vigitel Brasil 2014: vigilância de fatores de risco e proteção para doenças crônicas por inquérito telefônico. Brasília (DF); 2015 [citado 3 nov 2017]. Disponível em: http://pesquisa. bvsalud.org/bvsms/resource/pt/ mis-37130 pacientes sem rinite. É provável que isso se deva à maior gravidade da asma observada nos pacientes que não tinham essa associação na população estudada. Infecções respiratórias como sinusites, otites e pneumonias também são mais frequentes nesses pacientes ${ }^{6,7, a}$, o que contribui para os custos associados. O custo incremental relacionado à rinite ou IR foi de $11,7 \%$, com custo médio total relacionado à asma de $\mathrm{R} \$ 1.975,80 /$ paciente-ano $(\mathrm{DP}=\mathrm{R} \$ 2.232,55)$, alcançando valores de $\mathrm{R} \$ 2.207,99(\mathrm{DP}=\mathrm{R} \$ 2.220,55)$ para a situação de asma associada à rinite ou IR. Os custos diretos representaram a maior proporção e o custo com medicamentos quase a metade do custo da asma.

Na época da coleta de dados, 30,0\% dos pacientes obtinham a medicação de controle da asma gratuitamente no SUS, o que impactava diretamente o orçamento da maioria das famílias. O custo médio anual total teve impacto significativo na economia familiar, correspondendo a 11,6\% da RFM anual. Esse valor correspondeu a 9,0\% do produto interno bruto per capita brasileiro em 2012 . Em estudo que avaliou pacientes com asma grave na Bahia, o custo direto da doença correspondeu a $24,0 \%$ da RFM no ano anterior à inclusão dos pacientes em programa estruturado de asma. $\mathrm{O}$ fornecimento gratuito de medicamentos reduziu tal proporção para $2,0 \%$ da $\mathrm{RFM}^{13}$.

Quanto mais grave ou pior o controle da asma, maior é o seu custo ${ }^{1,18,27}$. Os custos da asma grave foram 128\%, 259\% e 253\% maiores em relação à asma moderada, leve e intermitente, respectivamente. Também foi maior na asma não controlada em comparação à parcialmente controlada $(>19,0 \%)$ e controlada ( $>37,0 \%)$. Proporcionalmente à RFM, asmáticos graves tiveram custo duas a quatro vezes maiores se comparados a pacientes com asma moderada, leve ou intermitente. Da mesma forma, asmáticos não controlados tiveram custos proporcionais a sua RFM quatro a seis vezes maiores do que pacientes parcialmente controlados e controlados. Pacientes com asma grave e moderada, bem como os não controlados, tiveram redução da RFM durante o estudo. Apenas entre asmáticos controlados ou leves, houve crescimento da RFM, apesar da pequena redução do PIB per capita brasileiro entre 2011 e $2012^{\mathrm{d}}$. Embora nosso estudo não tenha sido desenhado para isso, os achados sugerem que quanto mais grave ou pior o controle da asma, maior é sua influência sobre a capacidade de trabalho e produtividade das famílias, que parecem aumentar com o melhor controle da doença.

O custo da doença não controlada também foi maior em estudo que comparou os custos diretos do tratamento de pacientes com asma controlada e não controlada em unidade terciária em São Paulo ${ }^{22}$. Isso ocorreu principalmente porque os pacientes iam muito mais à emergência e eram hospitalizados com maior frequência, o que não aconteceu em nosso estudo. Mesmo assim, tivemos maior custo nesse subgrupo. Os pacientes não controlados também tinham renda familiar menor, como observamos na população estudada. Em um centro de referência para asma grave na Bahia, 74,0\% dos pacientes tinham RFM inferior ao salário mínimo ${ }^{21}$. O mesmo grupo mostrou redução importante dos custos diretos da asma grave depois de alcançado o controle da doença ${ }^{13}$.

Comorbidades são frequentes em adultos com asma e podem ter impacto nos seus custos ${ }^{14}$. O aumento de sobrepeso/obesidade nas sociedades ocidentais associa-se ao aumento da prevalência, gravidade e pior controle da asma ${ }^{5,11,19}$. Nossos resultados sinalizam para maiores custos em obesos do que em asmáticos com sobrepeso (>33,0\%) e com peso normal (> 76,0\%). Isso é particularmente preocupante considerando que, além da elevada prevalência de asma em nosso meio, os dados de 2012 da pesquisa Vigitel mostravam que 64,3\% dos adultos brasileiros tinham excesso de peso $(48,5 \%)$ ou obesidade $(15,8 \%)^{\text {e.f. }}$. Esses valores aumentaram para 70,4\%, 52,5\% e 17,9\%, respectivamente, em 2014. Programas estruturados para abordagem à asma associados a programas para combate ao sobrepeso ou obesidade poderão ser úteis em atenção primária para melhor controle da asma e redução de custos associados em nosso meio.

A asma pode ter consequências na capacidade de trabalho e o absenteísmo é frequentemente subestimado pelos formuladores de políticas públicas de saúde. $\mathrm{O}$ absenteísmo por doença é influenciado por variáveis demográficas, nível de satisfação no trabalho, características 
organizacionais da instituição e do conteúdo da atividade em questão ${ }^{17}$. Nossa população apresentava grande diversidade de ocupações e locais de trabalho, o que impede qualquer análise sobre esses fatores. Quase $80 \%$ dos trabalhadores neste estudo tiveram faltas ao trabalho e três quartos dos estudantes perderam dias de aula exclusivamente em função da asma. $\mathrm{O}$ custo anual total estimado relacionado com todas as licenças correspondeu a $17,7 \%$ do custo total anual estimado. O custo indireto foi baixo em comparação com outros estudos ${ }^{4}$, provavelmente devido a menor faixa de renda de nossos pacientes em relação a países desenvolvidos.

Nosso estudo possui limitações. A população estudada não representa a população de asmáticos brasileiros, mas sim, a população de asmáticos atendidos na unidade de saúde em questão. Os participantes correspondiam a um terço do total de asmáticos acompanhados e sua inclusão se deu consecutivamente para minimizar vieses de seleção. Outra limitação refere-se à ausência de dados confiáveis sobre a distribuição socioeconômica e as proporções de níveis de gravidade dos asmáticos brasileiros. Desta forma, a população estudada não representa necessariamente a realidade nacional. O tratamento recebido por esses pacientes não espelha a rotina dos serviços públicos, pois se trata de unidade secundária de uma universidade, que segue diretrizes internacionais recentes. Suas características estruturais e operacionais promoveram boa taxa de adesão ao tratamento e um número pequeno de hospitalizações, possivelmente subestimando o custo relacionado à doença.

O custo anual estimado de asma na população estudada teve impacto considerável sobre o orçamento familiar e foi maior em pacientes adultos, com mais de 20 anos de doença, em obesos ou com sobrepeso, na asma mais grave ou com pior controle. As unidades de custo com maior potencial de impacto no custo total foram os medicamentos para asma, as licenças prolongadas, as medidas de controle ambiental e os medicamentos para rinite.

Estudos de dimensionamento do impacto socioeconômico da asma podem fornecer maior embasamento para tomada de decisão aos formuladores de políticas de saúde, particularmente importante em um cenário de escassez de recursos. Nossos resultados são uma estimativa do custo do tratamento da asma em nível secundário no SUS, assumindo-se que o tratamento baseado na GINA represente a abordagem ideal da doença em nosso meio. Estudos com a mesma metodologia podem ser replicados em diferentes regiões e em unidades com níveis distintos de atenção à saúde, para expandir o conhecimento sobre seus reais custos no Brasil.

\section{REFERÊNCIAS}

1. Accordini S, Bugiani M, Arossa W, Gerzeli S, Marinoni A, Olivieri M, et al. Poor control increases the economic cost of asthma: multi-centre population-based study. Int Arch Allergy Immunol. 2006;141(2):189-98. https://doi.org/10.1159/000094898

2. Accordini S, Corsico AG, Braggion M, Gerbase MW, Gislason D, Gulsvik A, et al. The cost of persistent asthma in Europe: an international population-based study in adults. Int Arch Allergy Immunol. 2013;160(1):93-101. https://doi.org/10.1159/000338998

3. Araújo DV, Tavares LR, Veríssimo R, Ferraz MB, Mesquita ET. Cost of heart failure in the Unified Health System. Arq Bras Cardiol. 2005;84(5):422-7. https://doi.org/10.1590/S0066-782X2005000500013

4. Bahadori K, Doyle-Waters MM, Marra C, Lynd L, Alasaly K, Swiston J, et al. Economic burden of asthma: a systematic review. BMC Pulm Med. 2009; 9:24-39. https://doi.org/10.1186/1471-2466-9-24

5. Boulet LP. Asthma and obesity. Clin Exp Allergy. 2013;43(1):8-21. https://doi.org/10.1111/j.1365-2222.2012.04040.x

6. Bousquet J, Van Cauwenberge P, Khaltaev N; ARIA Workshop Group; World Health Organization. Allergic rhinitis and its impact on asthma (ARIA). J Allergy Clin Immunol. 2001;108(5 Suppl):147-334.

7. Bousquet J, Khaltaev N, Cruz AA, Denburg J, Fokkens WJ, Togias A, et al. Allergic Rhinitis and its Impact on Asthma (ARIA) update 2008. Allergy. 2008;63 Suppl 86:8-160. https://doi.org/10.1111/j.1398-9995.2007.01620.x 
8. Costa E, Bregman M, Araújo DV, Costa CH, Rufino R. Asthma and the socio-economic reality in Brazil. World Allergy Organ J. 2013;6(1):20. https://doi.org/10.1186/1939-4551-6-20

9. De Onis M, Onyango AW, Borghi E, Siyam A, Nishida C, Siekmann J. Development of a WHO growth reference for school-aged children and adolescents. Bull World Health Organ. 2007;85(9):660-7. https://doi.org/10.2471/BLT.07.043497

10. Fiori NS, Gonçalves H, Dumith SC, Cesar MADC, Menezes AMB, Macedo SEC. Ten-year trends in prevalence of asthma in adults in southern Brazil: comparison of two population-based studies. Cad Saude Publica. 2012;28(1):135-44. https://doi.org/10.1590/S0102-311X2012000100014

11. Ford ES. The epidemiology of obesity and asthma. J Allergy Clin Immunol. 2005;115(5):897-909. https://doi.org/10.1016/j.jaci.2004.11.050

12. Forno E, Gogna M, Cepeda A, Yañez A, Solé D, Cooper P, et al. Asthma in Latin America. Thorax. 2015;70(9):898-905. https://doi.org/10.1136/thoraxjnl-2015-207199

13. Franco R, Nascimento HF, Cruz AA, Santos AC, Souza-Machado C, Ponte EV, et al. The impact of severe asthma to low-income families. Allergy. 2009;64(3):478-83. https://doi.org/10.1111/j.1398-9995.2009.01981.x

14. Gergen PJ. Surveillance of the cost of asthma in the 21st century. I Allergy Clin Immunol. 2011;127(2):370-1. https://doi.org/10.1016/j.jaci.2010.11.023

15. Kuschnir FC, Gurgel RQ, Solé D, Costa E, Felix MMR, Oliveira CL, et al. ERICA: prevalence of asthma in Brazilian adolescents. Rev Saude Publica. 2016;50 Supl1:13s. https://doi.org/10.1590/S01518-8787.2016050006682

16. Lai CKW, Kim YY, Kuo S-H, Spencer M, Williams AE. Cost of asthma in the Asia-Pacific region. Eur Repir Rev. 2006;15:10-6. https://doi.org/10.1183/09059180.06.00009802

17. Mesa FR, Kaempffer AM. 30 anos de estudio sobre ausentismo laboral en Chile: una perspectiva por tipos de empresas. Rev Med Chile. 2004;132(9):1100-8. https://doi.org/10.4067/S0034-98872004000900012

18. O’Neill S, Sweeney J, Patterson CC, Menzies-Gow A, Niven R, Mnsur AH, et al. The cost of treating severe refractory asthma in UK: an economic analysis from the British Thoracic Society Difficult Asthma Registry. Thorax. 2015;70(4):376-8. https://doi.orgt/10.1136/thoraxjnl-2013-204114

19. Pawankar R, Baena-Cagnani CE, Bousquet JB, Canonica GW, Cruz AA, Kaliner MA, et al. State of World Allergy Report 2008: allergy and chronic respiratory diseases. World Allergy Organ J. 2008;1 Suppl 1:S4-17. https://doi.org/10.1097/1939-4551-1-S1-S1

20. Ribeiro RA, Mello RGB, Melchior R, Dill JC, Hohmann CB, Lucchese AM, et al. Annual cost of ischemic heart disease in Brazil: public and private perspective. Arq Bras Cardiol. 2005;85(1):3-8. https://doi.org/10.1590/S0066-782X2005001400002

21. Ponte E, Franco RA, Souza-Machado A, Souza-Machado C, Cruz AA. Impacto de um programa para o controle da asma grave na utilização de recursos do Sistema Único de Saúde. J Bras Pneumol. 2007;33(1):15-9. https://doi.org/10.1590/S1806-37132007000100006

22. Santos LA, Oliveira MA, Faresin SM, Santoro IL, Fernandes ALG. Direct cost of asthma in Brazil: a comparison between controlled and uncontrolled asthmatic patients. Braz I Med Biol Res. 2007;40(7):943-8. https://doi.org/10.1590/S0100-879X2006005000129

23. Sociedade Brasileira de Pneumologia e Tisiologia. Diretrizes para o manejo da asma 2012. J Bras Pneumol. 2012;38 Supl1:S1-46.

24. Solé D, Wandalsen GF, Camelo-Nunes IC, Naspitz CK; ISAAC - Brazilian Group. Prevalence of symptoms of asthma, rhinitis, and atopic eczema among Brazilian children and adolescents identified by the International Study of Asthma and Allergies in Childhood (ISAAC) Phase 3. J Pediatr (Rio J). 2006;82(5):341-6. http://dx.doi.org/10.2223/JPED.1521

25. Stirbulov R, Silva NL, Maia SCOM, Carvalho-Netto E, Angelini L. Cost of severe asthma in Brazil: systematic review. J Asthma. 2016;53(10):1063-70. Disponível em: https://doi.org/10.3109/02770903.2016.1171338

26. Sullivan PW, Ghushchyan VH, Slejko JF Belozeroff V, Globe DR, Lin SL. The burden of adult asthma in the United States: evidence from the Medical Expenditure Panel Survey. J Allergy Clin Immunol. 2011;127(2):363-9.e1-3. https://doi.org/10.1016/j.jaci.2010.10.042 
27. Sullivan SD, Rasouliyan L, Russo PA, Kamath T, Chipps BE; TENOR Study Group. Extent, patterns, and burden of uncontrolled disease in severe or difficult-to-treat asthma. Allergy. 2007;62(2):126-33. https://doi.org/10.1111/j.1398-9995.2006.01254.x

28. Van Den Akker-van Marle ME, Bruil J, Detmar SB. Evaluation of cost of disease: assessing the burden to society of asthma in children in the European Union. Allergy. 2005;60(2):140-9. https://doi.org/10.1111/j.1398-9995.2005.00692.x RC. Responsabilidade pública pelo conteúdo do artigo: EC.

Conflito de Interesses: Os autores declaram não haver conflito de interesses. 